\title{
Intra-Arterial Infusion of a Glycoprotein IIb/Illa Antagonist for the Treatment of Thromboembolism During Coil Embolization of Intracranial Aneurysm: A Comparison of Abciximab and Tirofiban
}

H.W. Jeong and S.-C. Jin

\begin{abstract}
BACKGROUND AND PURPOSE: Abciximab and tirofiban are commonly used for the treatment of thromboembolisms that form during coiling of intracranial aneurysms; however, it is not known which of these inhibitors is safer and more effective. We report the safety and the recanalization rates for intra-arterial abciximab and intra-arterial tirofiban infusion for the treatment of thromboembolisms that form during coiling.
\end{abstract}

MATERIALS AND METHODS: Between March 2004 and April 2011, 346 intracranial aneurysms were treated with coiling. Thromboembolisms developed in 22 of these patients and were treated by use of intra-arterial tirofiban $(n=11)$ or abciximab $(n=11)$ infusion.

RESULTS: In the abciximab group, the thromboembolisms were completely $(n=1)$ or partially $(n=7)$ resolved in 8 cases $(72.7 \%)$ at the time of the final control angiography. Complete $(n=9)$ or partial $(n=2)$ resolution was achieved in all cases at the time of follow-up angiography ( $<3$ days after the procedure). In the tirofiban group, thromboembolisms were completely $(n=4)$ or partially $(n=6)$ resolved in 10 cases $(90.9 \%)$ at the time of the final control angiography. Complete $(n=9)$ or partial $(n=2)$ resolution was observed in all cases at the time of the follow-up angiography. There were no statistically significant differences between the 2 groups with respect to thrombus resolution (final angiography, $P=.311$; follow-up angiography, $P=.707$ ). No hemorrhagic complications developed in either group.

CONCLUSIONS: These results suggest that tirofiban is more effective than abciximab for the immediate resolution of thromboembolisms, with no statistical significance. Both intra-arterial tirofiban and abciximab exhibited similar safety and recanalization rates.

ABBREVIATIONS: GOS = Glasgow Outcome Scale; $\mathrm{HH}=$ Hunt and Hess

G lycoprotein IIb/IIIa receptors play a key role in maintaining physiologic hemostasis and in pathologic thrombus formation. ${ }^{1}$ Indeed, glycoprotein IIb/IIIa is often termed the final common component of platelet aggregation. Several glycoprotein IIb/ IIIa inhibitors, such as abciximab, eptifibatide, and tirofiban, have been used recently to treat patients with acute coronary syndrome. Abciximab and eptifibatide are more potent platelet aggregation inhibitors than is tirofiban. ${ }^{2-4}$ Therefore, abciximab is more commonly used than tirofiban in medical practice to treat acute coronary syndrome. However, when treating thromboembolisms that develop during coil embolization of cerebral aneu-

Received September 12, 2012; accepted after revision November 15

From the Department of Diagnostic Radiology (H.W.J.), Busan Paik Hospital, Inje University, Busan, Korea; and Department of Neurosurgery (S.-C.J.), Inje University Haeundae Paik Hospital, Busan, Korea.

This study was supported by a 2012 research grant from Inje University.

Please address correspondence to Sung-Chul Jin, MD, Department of Neurosurgery, Inje University Haeundae Paik Hospital, 1435 Jwa-dong Haeundae-gu, Busan 612-043, Republic of Korea; e-mail: kusmal@hanmail.net

-w. Indicates open access to non-subscribers at www.ajnr.org

http://dx.doi.org/10.3174/ajnr.A3501 rysms, abciximab can induce intracerebral hemorrhages, which can be fatal. ${ }^{5}$ Accordingly, tirofiban has been used recently instead of abciximab, on the basis of experience. However, studies comparing abciximab and tirofiban for the treatment of thromboembolisms that develop during coil embolization of cerebral aneurysms have not been published. We randomly used either intra-arterial abciximab or tirofiban without a loading dose to treat such thromboembolisms.

This study compared the safety and efficacy of intra-arterial abciximab infusion and intra-arterial tirofiban infusion for the treatment of thromboembolisms that formed during coil embolization of cerebral aneurysms.

\section{MATERIALS AND METHODS}

From March 2004 to April 2011, 346 aneurysms were treated with coil embolization at our institution. Thromboembolic insults requiring thrombolysis developed during 22 procedures ( 15 unruptured and 7 ruptured cerebral aneurysms). The thromboembolisms were confirmed by procedural control angiography.

The thromboembolisms were treated using intra-arterial ab- 
Table 1: Summary of anatomic and clinical data of the abciximab group

\begin{tabular}{|c|c|c|c|c|c|c|c|c|c|c|}
\hline Sex & $\begin{array}{l}\text { Age } \\
\text { (y) }\end{array}$ & $\begin{array}{l}\mathrm{HH} \\
\text { Grade }\end{array}$ & Ruptured & Aneurysm Location & $\begin{array}{l}\text { Size } \\
(\mathrm{mm})\end{array}$ & Affected Vessel & $\begin{array}{l}\text { Affected Vessel } \\
\text { Flow }\end{array}$ & $\begin{array}{l}\text { Dose } \\
(\mathrm{mg})\end{array}$ & Final Angiography & $\begin{array}{l}\text { Follow-Up } \\
\text { Angiography }\end{array}$ \\
\hline Male & 64 & 4 & ० & Left AcomA & $8.6 \times 4.1$ & Left A2 & Preserved & 15 & No change & Complete resolution \\
\hline Female & 60 & 3 & o & Right AchA & $9.9 \times 4.8$ & Right distal ICA & Preserved & 10 & No change & Complete resolution \\
\hline Male & 41 & 3 & ० & Basilar top & $3.5 \times 5.0$ & Both P1 & Preserved & 10 & Partial resolution & Complete resolution \\
\hline Female & 75 & 0 & $x$ & Right AcomA & $3.4 \times 2.9$ & Right A2 & Preserved & 10 & Partial resolution & Complete resolution \\
\hline Male & 61 & 0 & o & Right pericallosal & $5.0 \times 4.0$ & Right callosomarginal & Preserved & 10 & Partial resolution & Complete resolution \\
\hline Male & 54 & 0 & $x$ & Right SCA & $2.6 \times 2.2$ & Both SCA & Decreased & 20 & Partial resolution & Partial resolution \\
\hline Male & 53 & 2 & ० & Right AcomA & $8.3 \times 5.2$ & Right A2 & Preserved & 10 & Partial resolution & Complete resolution \\
\hline Male & 44 & 0 & $x$ & Basilar top & $4.8 \times 4.5$ & Left P1 & Arrested & 10 & No change & Complete resolution \\
\hline Male & 44 & 0 & $x$ & Left paraclinoid & $3.6 \times 3.6$ & Left A2 & Arrested & 20 & Complete resolution & Complete resolution \\
\hline Female & 67 & 1 & $\mathrm{x}$ & Left ICA bifurcation & $12 \times 12$ & Left Ml & Arrested & 10 & $\begin{array}{l}\text { M1 partial resolution } \\
\text { M3 distal migration }\end{array}$ & $\begin{array}{l}\text { M1 complete resolution } \\
\text { M3 no change }\end{array}$ \\
\hline Female & 65 & 0 & $x$ & Left SCA & $10.1 \times 8.1$ & Left SCA & Decreased & 10 & Partial resolution & Partial resolution \\
\hline
\end{tabular}

Note:-AcomA indicates anterior communicating artery; AchA, anterior choroidal artery; SCA, superior cerebellar artery.

Table 2: Summary of anatomic and clinical data of the tirofiban group

\begin{tabular}{|c|c|c|c|c|c|c|c|c|c|c|}
\hline Sex & $\begin{array}{l}\text { Age } \\
\text { (y) }\end{array}$ & $\begin{array}{l}\mathrm{HH} \\
\text { Grade }\end{array}$ & Ruptured & Aneurysm Location & $\begin{array}{l}\text { Size } \\
(\mathrm{mm})\end{array}$ & Affected Vessel & $\begin{array}{l}\text { Affected } \\
\text { Vessel } \\
\text { Flow }\end{array}$ & $\begin{array}{l}\text { Dose } \\
\text { (mg) }\end{array}$ & Final Angiography & Follow-Up Angiography \\
\hline Female & 46 & 0 & $x$ & Right SCA & $4.7 \times 4.2$ & Right SCA & Arrested & 0.2 & Complete resolution & Complete resolution \\
\hline Female & 67 & 1 & $x$ & Left paraclinoid & $3.8 \times 4.0$ & Left M4 (angular branch) & Arrested & 0.2 & No change & Partial resolution \\
\hline Female & 80 & 0 & $x$ & Left ICA bifurcation & $4.5 \times 7.0$ & Left $M 2$, left proximal $A 1$ & Decreased & 0.6 & Partial resolution & Complete resolution \\
\hline Female & 61 & 0 & $x$ & Right SCA & $3.9 \times 4.0$ & Right SCA & Decreased & 1.0 & Partial resolution & Partial resolution \\
\hline Female & 40 & 2 & $x$ & AcomA & $5.2 \times 3.4$ & Left proximal A2 & Preserved & 0.3 & Partial resolution & Complete resolution \\
\hline Male & 33 & 2 & ० & AcomA & $7.0 \times 5.5$ & Left A2 & Arrested & 0.2 & Complete resolution & Complete resolution \\
\hline Female & 44 & 2 & $x$ & Left AchA & $9.1 \times 7.0$ & Left M1 & Arrested & 1.0 & $\begin{array}{l}\text { M1 complete resolution } \\
\text { M3 distal migration }\end{array}$ & $\begin{array}{l}\text { M1 complete resolution } \\
\text { M3 no change }\end{array}$ \\
\hline Female & 65 & 0 & $x$ & AcomA & $8.0 \times 6.5$ & Left A2 & Preserved & 0.3 & Complete resolution & Complete resolution \\
\hline Female & 68 & 0 & $x$ & Right AchA & $3.5 \times 3.1$ & Right AchA & Decreased & 0.5 & Partial resolution & Complete resolution \\
\hline Female & 71 & 1 & $x$ & Right pericallosal & $3.3 \times 1.9$ & Right callosomarginal & Preserved & 0.4 & Partial resolution & Complete resolution \\
\hline Male & 50 & 2 & o & Right proximal A1 & $3.0 \times 4.2$ & Right proximal $\mathrm{A} 1$ & Decreased & 0.5 & Partial resolution & Complete resolution \\
\hline
\end{tabular}

Note:-SCA indicates superior cerebellar artery; AcomA, anterior communicating artery; AchA, anterior choroidal artery.

ciximab $(n=11$, Table 1$)$ or intra-arterial tirofiban $(n=11$, Table 2 ), without any adjuvant fibrinolytic or anti-platelet medication.

Bare platinum coils were used in all coil embolizations of cerebral aneurysms. Coil embolization by use of single-catheter, double-catheter, stent-assisted, or balloon-assisted techniques was performed for thromboembolisms that were treated with either intra-arterial abciximab or intra-arterial tirofiban infusion (abciximab group: single microcatheter, 8 cases; double microcatheter, 1 case; and stent-assisted, 2 cases; tirofiban group: single microcatheter, 8 cases; double microcatheter, 1 case; balloon-assisted, 1 case; and stent-assisted, 1 case).

Thromboembolism requiring thrombolysis was defined as either the gradual progression of a thrombus or an antegrade flow disturbance produced by the thrombus, as visualized by control procedural angiography. The location of the thrombi was divided into the proximal thrombus (coil/parent artery interface) and the distal thrombus (distal to the aneurysm).

Angiographic thrombotic occlusion was graded according to the antegrade flow disturbance distal to the thrombus as occlusive, partial, or preserved antegrade flow. Angiographic recanalization was classified as complete recanalization, partial recanalization, or unchanged flow after thrombolysis.

A brain CT was usually performed within several hours after the procedure to evaluate the extent of cerebral infarction or hemorrhage. Conventional angiography was repeatedly performed to evaluate the degree of thrombus resolution within 3 days after the procedure.

The clinical severity of patients with subarachnoid hemorrhage was classified by use of the Hunt and Hess scale, and the clinical outcome at discharge was assessed with the Modified Glasgow Outcome Scale. The GOS scores were dichotomously classified as good (GOS score, 4-5) or poor (GOS score, 1-3) functional outcomes. ${ }^{6}$

\section{Procedure}

To minimize thromboembolic insults induced by embolization, anticoagulation was initiated by the injection of a bolus of $4000 \mathrm{IU}$ heparin intravenously immediately after femoral puncture. This injection was followed by intermittent bolus injections of $1000 \mathrm{IU}$ heparin hourly to maintain the activated coagulation time at approximately twice the baseline level. Before stent-assisted coiling, we administered aspirin (100 mg) and clopidogrel $(75 \mathrm{mg})$ to the patients with unruptured cerebral aneurysms for at least 7 days before the procedure. For patients with ruptured cerebral aneurysms, $300 \mathrm{mg}$ of aspirin and clopidogrel was administered immediately after the procedure through a nasogastric tube.

The treatment strategy for thromboembolism during the coil embolization of cerebral aneurysms consisted of intra-arterial abciximab or intra-arterial tirofiban infusion, without adjuvant fibrinolytic or antiplatelet medications during the procedure. Intermittent hand injections of intra-arterial abciximab (typical dose: $10-20 \mathrm{mg}, 2 \mathrm{mg}$ at a time) or intra-arterial tirofiban (typical dose: $100-1000 \mu \mathrm{g}, 50 \mu \mathrm{g}$ at a time) without a loading dose were performed near the thrombus according to the degree of thrombus resolution. Infusion was ceased once arterial flow was restored or when we reached the upper dose limits (abciximab: 20 mg; tirofiban: $1000 \mu \mathrm{g}$ ).

\section{Statistical Analysis}

Nominal data were analyzed with the $\chi^{2}$ test or Fisher exact test, and numeric data were analyzed with Student $t$ test or the Mann-Whitney $U$ test as appropriate. The results of 2-tailed 

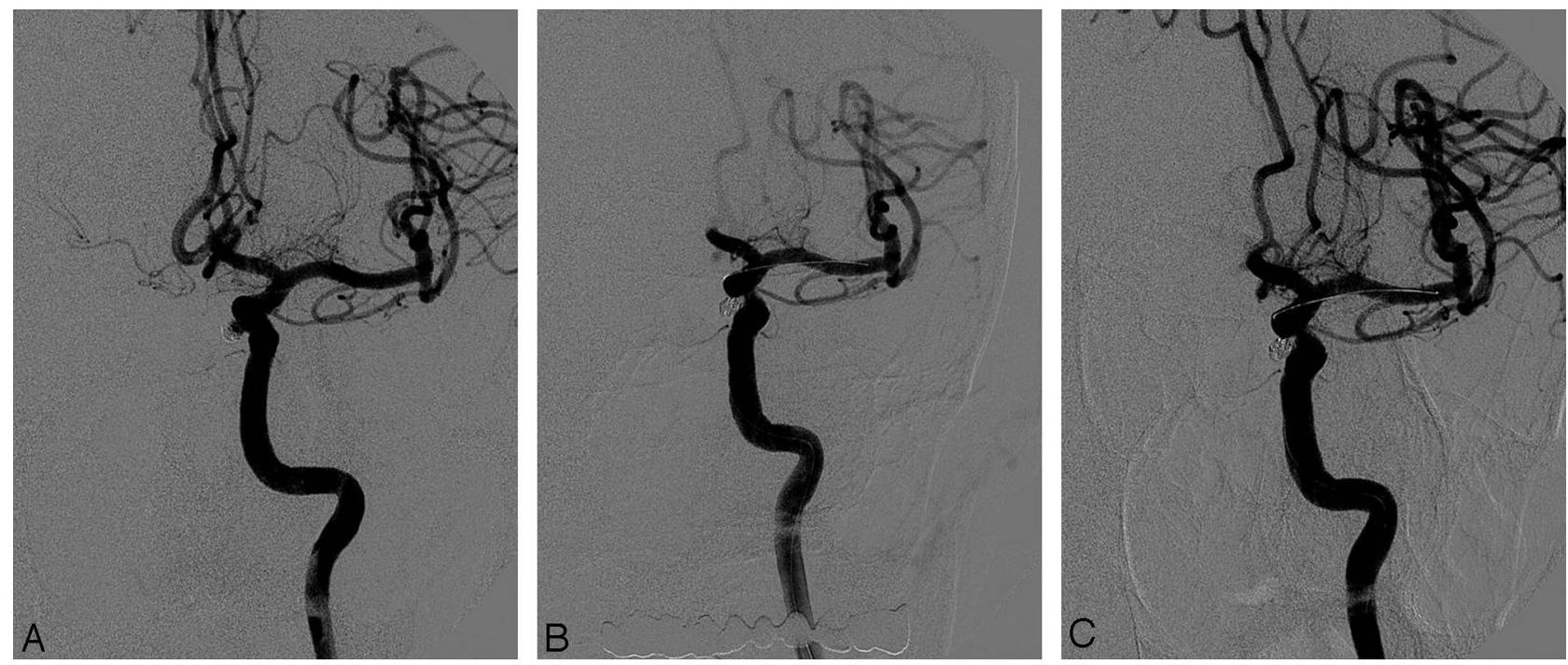

FIG 1. A 44-year-old man. A, Pre-embolization angiogram showing a recanalized left paraclinoid aneurysm. $B$, Postembolization angiogram showing the well-occluded aneurysm with 1 coil and arrested flow in the A2 segment of the left anterior cerebral artery. C, Final angiogram showing complete recanalization of the occluded branch after superselective intra-arterial infusion of abciximab (20 mg).
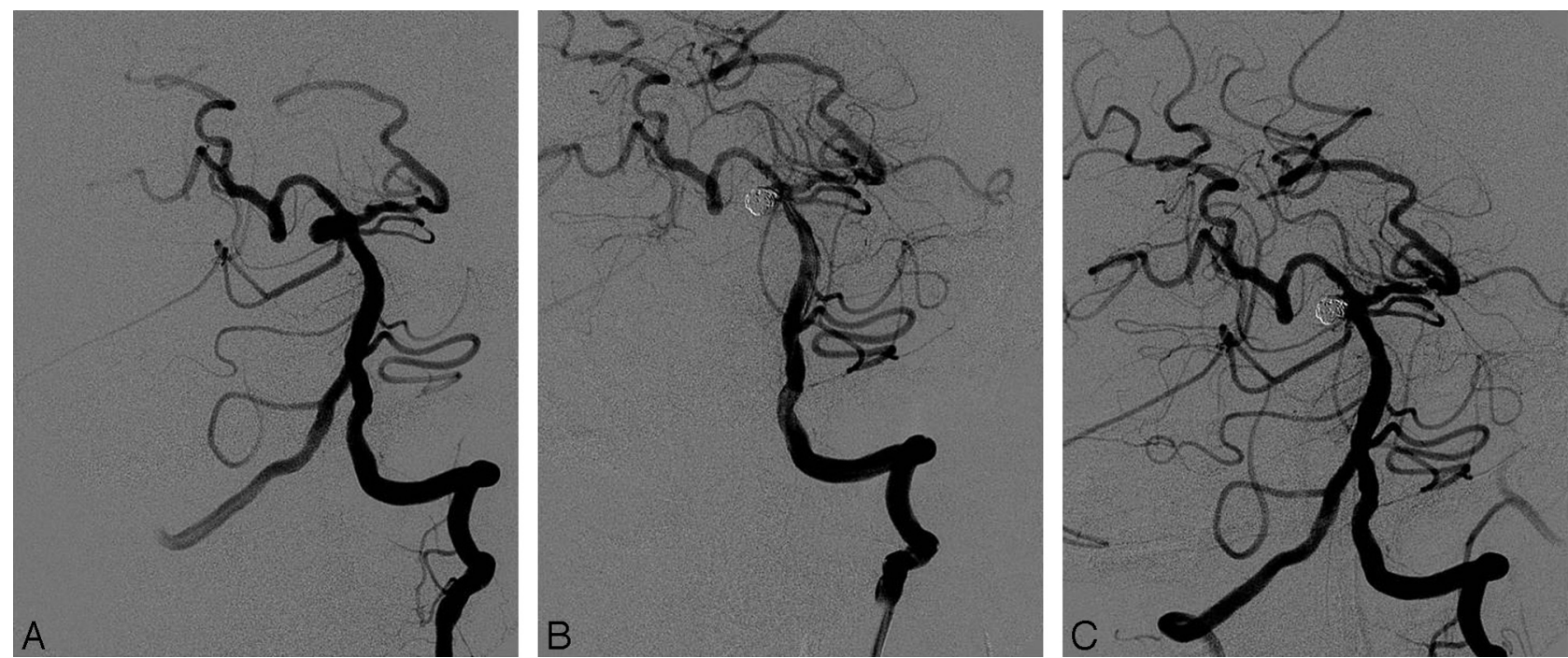

FIG 2. A 46-year-old woman. A, Pre-embolization angiogram showing a saccular aneurysm at the origin of the right SCA. B, Postembolization angiogram showing the well-occluded aneurysm with 3 detachable coils and occlusion of the right SCA. C, Final angiogram showing complete recanalization of the occluded right SCA after superselective intra-arterial infusion of tirofiban (0.2 mg).

tests were considered statistically significant at the $P<.05$ level.

\section{RESULTS}

Twenty-two patients with cerebral aneurysm and thromboembolism were treated with intra-arterial abciximab or tirofiban infusion. The location of the aneurysm was the anterior cerebral artery in 9 patients (anterior communicating artery: $n=3$; proximal anterior cerebral artery: $n=1$; distal anterior cerebral artery: $n=$ $5)$, the internal carotid bifurcation in 2 patients, the anterior choroidal artery in 3 patients, the paraclinoid carotid artery in 2 patients, and the basilar bifurcation in 6 patients (Tables 1 and 2).

A proximal thrombus at the coil/parent artery interface was identified in 18 cases in the following categories: 1) occlusion of the affected vessel in 4 cases, 2) partial flow of the affected vessel in
6 cases, and 3) preserved flow of the affected vessel in 8 cases. A distal thrombus was detected in 4 cases; occlusion of the affected vessel occurred in 3 cases, and partial flow of the affected vessel was found in 1 case.

In the abciximab-treated group, final control angiography revealed complete $(n=1)$ or partial recanalization $(n=7)$ in 8 cases (72.7\%) (Fig 1). Follow-up angiography revealed complete $(n=$ $9)$ or partial recanalization $(n=2)$ in all cases. In the tirofibantreated group, final control angiography revealed complete $(n=$ 4) or partial recanalization ( $n=6)$ in 10 cases (90.9\%) (Fig 2), and follow-up angiography revealed complete $(n=9)$ or partial recanalization $(n=2)$ in all cases. There were no instances of cerebral hemorrhage related to the procedure in either group. One patient in the abciximab-treated group had neurologic deficits related to the vascular area affected by the thromboembolism 
(GOS score, 3). There was no statistically significant difference in the rate of aneurysm rupture, the thrombus location, or the degree of angiographic thrombotic occlusion between the abciximab-treated and tirofiban-treated groups. There was no statistically significant difference in the immediate recanalization status between groups.

\section{DISCUSSION}

Thromboembolism is a major complication during all steps of coil embolization because thromboembolisms disrupt blood flow and may cause intimal injuries. Accordingly, the prevention and treatment of thromboembolisms are major issues relevant to the use of coil embolization for cerebral aneurysms. Several agents, such as fibrinolytic drugs, tirofiban, and abciximab, have been used intravenously or intra-arterially to treat thromboembolisms during coil embolization of cerebral aneurysms. ${ }^{5,7-12}$ The general consensus is that fibrinolytic agents should not be used for thrombolysis because they carry a significant risk of cerebral hemorrhage. The use of abciximab for thromboembolism during coil embolization of cerebral aneurysms remains controversial; some studies have reported that it is associated with cerebral hemorrhage and severe thrombocytopenia, whereas others have reported no such relationship. ${ }^{5,7,8}$ To reduce or prevent these problems, tirofiban, which is a relatively short-acting and reversible glycoprotein IIb/IIIa receptor inhibitor, has been used with increasing frequency for the treatment of thromboembolisms during the coil embolization of cerebral aneurysms. Although there have been studies conducted for acute coronary syndrome, there have been no comparative studies investigating abciximab and tirofiban infusion for the treatment of thromboembolisms induced by coil embolization of cerebral aneurysms. Although our study was not a randomized, controlled study, our comparison of abciximab and tirofiban provides valuable information regarding the treatment of thromboembolisms that occur during coil embolization of cerebral aneurysms because there was no selection intent between abciximab and tirofiban.

The dosage and method of administration of abciximab or tirofiban for the treatment of thromboembolisms during coil embolization of cerebral aneurysms were entirely based on the standard application of these drugs for acute coronary syndrome. Therefore, the dose and application method of these drugs must be adjusted for acute-onset thromboembolisms, which may occur during coil embolization of cerebral aneurysms. In our study, to avoid procedural complications such as cerebral hemorrhage and to reach a high recanalization rate, we administered these drugs intra-arterially rather than intravenously. Because intra-arterial access is most proximal to the thrombus, a smaller total dose of the drug is expected to be effective, and, as a result, dose-dependent complications such as cerebral hemorrhage may be reduced. Additionally, we did not use a loading infusion of abciximab or tirofiban. Repeated intra-arterial pulsatile injections of small doses (abciximab: $2 \mathrm{mg}$; tirofiban: $50 \mu \mathrm{g}$ ) of the drugs may be sufficient to resolve a thrombus. In our series, the mean dose of abciximab was $12.2 \pm 4.1 \mathrm{mg}$, which represented approximately $70 \%$ of the recommended loading dose of abciximab (loading dose: $0.25 \mathrm{mg} / \mathrm{kg}$ ) for a $70-\mathrm{kg}$ patient. The minimal dose of abciximab was $10 \mathrm{mg}$, which exceeds $50 \%$ of the recommended loading dose. In comparison with our dose of abciximab, the mean dose of tirofiban was $470 \pm 290 \mu \mathrm{g}$, which represented approximately $56 \%$ of the recommended loading of tirofiban (loading dose: 0.4 $\mu \mathrm{g} / \mathrm{kg} / \mathrm{min} \times 30$ minutes) for a $70-\mathrm{kg}$ patient. Six patients were treated with $<50 \%$ of the recommended loading dose of tirofiban. Even though our data did not provide the weight of the patients, our doses of abciximab and tirofiban may be lower than usual loading doses. Our dose of tirofiban may be relatively lower than that of abciximab, on the basis of usual loading doses of tirofiban and abciximab. Accordingly, regarding cost-effectiveness and safety, the lower dose of tirofiban that was used may be more beneficial.

Our immediate and follow-up recanalization rates for intraarterial abciximab $(72.7 \%, 100 \%)$ and tirofiban $(90.9 \%, 100 \%)$ appear to be similar or superior to those reported in previous studies. ${ }^{5,7,8,10-12}$ This result may be related to the fact that these drugs were used to treat freshly formed thrombi, which may be more readily resolved. Therefore, we suggest that a loading dose of abciximab or tirofiban is not necessary for the treatment of thromboembolisms that form during the coil embolization of cerebral aneurysms.

In our series, both abciximab-treated and tirofiban-treated groups showed no hemorrhage in the ruptured cerebral aneurysms. This may be related to the relatively lower doses of abciximab or tirofiban in comparison with the usual doses.

In our study, the immediate recanalization rate of the tirofiban-treated group was superior to that of the abciximab-treated group, but this difference was not statistically significant. The follow-up recanalization rate was $100 \%$ in both groups, with no cerebral hemorrhages. Unlike in the treatment of acute coronary syndromes, ${ }^{2,3}$ the efficacy and safety of tirofiban may not be inferior to those of abciximab for the treatment of thromboembolisms during coil embolization of cerebral aneurysms. Accordingly, tirofiban, which has a short plasma half-life and is a competitive inhibitor of glycoprotein IIb/IIIa, may be more suitable than abciximab to treat thromboembolisms during coil embolization of cerebral aneurysms. Operations such as decompressive craniectomy or external ventricular drainage are sometimes necessary after coil embolization, especially in cases of ruptured cerebral aneurysms. Therefore, tirofiban is less likely than abciximab to induce operation-related hemorrhagic complications.

\section{CONCLUSIONS}

In our study, intra-arterial abciximab and tirofiban exhibited similar safety levels and did not induce procedure-related cerebral hemorrhages. In addition, the efficacy of tirofiban was similar or superior to that of abciximab; however, this difference was not statistically significant. Therefore, tirofiban may be more suitable for the treatment of thromboembolisms induced by coil embolization of cerebral aneurysms, especially in cases of ruptured cerebral aneurysms.

\section{REFERENCES}

1. Fiehler J, Ries T. Prevention and treatment of thromboembolism during endovascular aneurysm therapy. Klinische Neuroradiologie 2009;19:73-81

2. Batchelor WB, Tolleson TR, Huang Y, et al. Randomized comparison of platelet inhibition with abciximab, tirofiban and eptifibatide 
during percutaneous coronary intervention in acute coronary syndromes: the COMPARE trial. Comparison Of Measurements of Platelet aggregation with Aggrastat, Reopro, and Eptifibatide. Circulation 2002;106:1470-76

3. Herrmann HC, Swierkosz TA, Kapoor S, et al. Comparison of degree of platelet inhibition by abciximab versus tirofiban in patients with unstable angina pectoris and non-Q-wave myocardial infarction undergoing percutaneous coronary intervention. Am J Cardiol 2002;89:1293-97

4. Roe MT, Granger CB, Puma JA, et al. Comparison of benefits and complications of hirudin versus heparin for patients with acute coronary syndromes undergoing early percutaneous coronary intervention. Am J Cardiol 2001;88:1403-06, A1406

5. Park JH, Kim JE, Sheen SH, et al. Intraarterial abciximab for treatment of thromboembolism during coil embolization of intracranial aneurysms: outcome and fatal hemorrhagic complications. J Neurosurg 2008;108:450-57

6. Teasdale G, Jennett B. Assessment of coma and impaired consciousness: a practical scale. Lancet 1974;2:81-84

7. Ries T, Siemonsen S, Grzyska U, et al. Abciximab is a safe rescue therapy in thromboembolic events complicating cerebral aneu- rysm coil embolization: single center experience in $\mathbf{4 2}$ cases and review of the literature. Stroke 2009;40:1750-57

8. Aggour M, Pierot L, Kadziolka K, et al. Abciximab treatment modalities for thromboembolic events related to aneurysm coiling. $\mathrm{Neu}$ rosurgery 2010;67:503-08

9. Cronqvist $\mathrm{M}$, Pierot L, Boulin A, et al. Local intraarterial fibrinolysis of thromboemboli occurring during endovascular treatment of intracerebral aneurysm: a comparison of anatomic results and clinical outcome. AJNR Am J Neuroradiol 1998;19:157-65

10. Bruening R, Mueller-Schunk S, Morhard D, et al. Intraprocedural thrombus formation during coil placement in ruptured intracranial aneurysms: treatment with systemic application of the glycoprotein IIb/IIIa antagonist tirofiban. AJNR Am J Neuroradiol 2006;27:1326-31

11. Kang HS, Kwon BJ, Roh HG, et al. Intra-arterial tirofiban infusion for thromboembolism during endovascular treatment of intracranial aneurysms. Neurosurgery 2008;63:230-38

12. Song JK, Niimi Y, Fernandez PM, et al. Thrombus formation during intracranial aneurysm coil placement: treatment with intra-arterial abciximab. AJNR Am J Neuroradiol 2004;25:1147-53 